\title{
Expérience de dialogue entre l'anthropologie et une situation de développement au Vietnam
}

De la position d'observateur à celle de médiateur

\section{Emmanuel Pannier et Christian Culas}

\section{(2) OpenEdition Journals}

Édition électronique

URL : http://journals.openedition.org/anthropodev/511

DOI : 10.4000/anthropodev. 511

ISSN : 2553-1719

Éditeur

APAD - Association pour l'anthropologie du changement social et du développement

\section{Édition imprimée}

Date de publication : 1 juillet 2016

Pagination : 123-148

ISBN : 797-10-93476-03-2

ISSN : 2276-2019

\section{Référence électronique}

Emmanuel Pannier et Christian Culas, «Expérience de dialogue entre l'anthropologie et une situation de développement au Vietnam », Anthropologie \& développement [En ligne], 44 | 2016, mis en ligne le 01 janvier 2017, consulté le 01 mai 2019. URL : http://journals.openedition.org/anthropodev/511 ; DOI : 10.4000/anthropodev.511

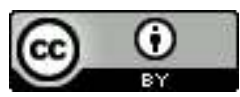

La revue Anthropologie \& développement est mise à disposition selon les termes de la Licence Creative Commons Attribution 4.0 International. 


\title{
Expérience de dialogue entre l'anthropologie et une situation de développement au Vietnam
}

\section{De la position d'observateur à celle de médiateur}

\author{
Emmanuel Pannier et Christian Culas ${ }^{\prime}$
}

Cet article présente l'expérience et les changements de postures de deux anthropologues qui ont exploré l'idée que, pour être efficaces, les projets de développement devraient être construits à partir d'enquêtes qualitatives rigoureuses, sans préjuger ni des manques locaux, ni du secteur, ni des modalités d'intervention. Afin de vérifier l'intérêt et l'efficacité de cette démarche, les auteurs l'ont concrètement mise en œuvre dans une commune Tay du nord du Vietnam. Pendant trois ans, une étude anthropologique d'approche classique a ainsi été menée dans cette localité. Elle a permis de dresser un diagnostic de la situation locale et d'évaluer les possibilités d'initier une action de développement. Elle a en particulier révélé qu'il n'était pas pertinent de lancer un projet supplémentaire dans cette commune. La seule action possible était celle de médiateur ou d'intermédiaire entre l'Etat - à l'origine des actions de développement - et les populations locales, afin de favoriser une meilleure adéquation de l'intervention avec les réalités du terrain. A la demande des autorités locales, les auteurs se sont alors engagés dans le montage d'un projet de développement touristique en train de voir le jour dans la commune. Ainsi, d'une position d'observateurs des projets ils sont passés à celle de médiateurs entre les paysans, les autorités locales et les agences de tourisme.

\footnotetext{
${ }^{1}$ Nous remercions Paul Sorrentino ainsi que les deux relecteurs anonymes pour leurs commentaires et suggestions.
} 
This paper describes the experience and change of position of two anthropologists who explored the idea that, in order to be more efficient, development projects should be based on long and rigorous qualitative surveys, without prejudging either local deficiencies, the sector of activity or the methods of intervention. In order to verify, and possibly demonstrate, the value and effectiveness of this approach, the authors have put it into practice in a Tay ethnic commune in northern Vietnam. For a period of three years a classical anthropological study was carried out in these villages. This survey allowed the authors to establish a diagnosis of the local situation and to evaluate the possibilities for initiating a development action. The study specifically revealed that it wasn't relevant to start a new project in this commune. The most suitable action was to act as mediator or intermediary between the State - which is responsible for development action - and the local population in order to foster a better match of the intervention with the reality on the ground. At the local authorities' request, the authors became involved in the setting up of a tourism development project already in its infancy in the commune. Thus, from being observers of development projects, they became the mediator between farmers, local authorities and tourist agencies.

\section{Introduction}

Les anthropologues sont depuis longtemps sollicités dans des dispositifs de changement social volontaires et impulsés de l'extérieur, que ce soit pour servir la "mission civilisatrice " à l'époque coloniale ou pour conseiller certains porteurs de projets de développement actuels. Ils peuvent alors intervenir comme promoteurs du développement, comme analystes critiques des actions menées, comme experts ou consultants, ou encore comme observateurs extérieurs qui prennent le développement pour objet de recherche. Leur participation oscille donc entre la prise de distance et l'engagement (Atlani-Duault et Vidal, 2009). Ainsi il est aujourd'hui communément admis dans la sphère du développement que les sciences sociales ont leur rôle à jouer dans 
les projets, notamment pour aider à cerner et prendre en compte les éléments " socioculturels " (Olivier de Sardan, 1995 ; Cernea, 1999; Guichaoua et Goussault, 2005). Cet état d'esprit s'est exprimé dès la fin des années 1940-1950, surtout dans le monde anglo-saxon, puis s'est généralisé dans les années 1970 (AtlaniDuault et Vidal, 2009). Le recrutement d'anthropologues dans les grandes institutions de développement (Banque mondiale, PNUD, USAID, etc.) est également significatif de cette tendance.

Néanmoins, le dialogue entre développeurs et anthropologues reste encore difficile (Guichaoua et Majeres, 1982; Lavigne Delville, 1997; Arditi, 2005), bien que les exemples de collaborations fructueuses soient de plus en plus fréquents (AtlaniDuault, 2007 ; Lavigne Delville, 2008 ; Bouju, 2011). Il convient ainsi de distinguer les avancées faites par les anthropologues du développement qui ont dépassé l'opposition développement/sciences sociales (Geronimi et al., 2007 ; Atlani-Duault et Vidal, 2009 ; Cahiers d'études africaines, 2011), les discours des bailleurs et opérateurs du développement qui reconnaissent volontiers le rôle des sciences sociales dans les programmes de développement, et les pratiques réelles de collaboration entre les développeurs et les anthropologues en tant que chercheurs et non pas consultants (Olivier de Sardan, 2011). La littérature sur la question indique que cette dernière est bien effective mais reste un défi majeur (Lavigne Delville, 2008).

$\mathrm{Au}$ Vietnam, si les chercheurs locaux et internationaux sont largement sollicités pour conseiller les responsables des politiques publiques et des projets de développement, l'anthropologie du développement en tant que discipline indépendante des projets reste très marginale (Rambo et al., 1995; Fforde, 2009). La recherche académique vietnamienne en sciences sociales est officiellement un outil au service de l'Etat et du développement. Mais, sauf en de rares exceptions, les études et expertises des chercheurs nationaux ne comportent aucune proposition qui 
$s^{\prime}$ oppose aux lignes officielles ${ }^{2}$. Dans les faits, en tant que fonctionnaires " au service de la Révolution » (Fontanel, 2001), ils peuvent difficilement infléchir les politiques et leurs idéologies sous-jacentes, même si certains d'entre eux profitent de leur position politique et de leurs réseaux pour orienter, en interne, leur mise en œuvre sur le terrain (Hannah, 2007).

De plus, dans le contexte vietnamien, l'Etat est impliqué comme responsable et opérateur local dans tous les programmes de développement, nationaux ou étrangers. II en résulte que les actions restent toujours plus ou moins dans sa logique, en tout cas sous son contrôle, même si localement on observe régulièrement des accommodements entre les directives du pouvoir central et les réalités locales (Fforde, 2009). De manière générale, les projets de développement au Vietnam sont top-down, volontaristes et technicistes. La population locale est formellement consultée et étudiée mais ses opinions, ses besoins et ses capacités ne sont quasiment pas pris en compte dans la conception des projets. S'ils sont intégrés, c'est pour voir comment mettre en œuvre, sur le terrain, les objectifs et les activités prédéfinis.

Une autre dimension à prendre en compte est le nombre considérable de projets de développement qui se déploient en milieu rural. Certaines communes "ethniques ${ }^{3}$ " des régions montagneuses en reçoivent plus de trois par an. On observe souvent de la part des autorités locales et des villageois des signes de lassitude devant ce que nous qualifions de "routine des projets ", provoquée par les nombreuses réunions de consultation formelle avec présence obligatoire ou par le travail qu'ils doivent fournir afin d'afficher le côté participatif. Cela ne les empêche pas de tirer profit de l'intervention ou bien de l'éviter si celle-ci présente trop de contraintes. En bref, dans ce contexte spécifique,

\footnotetext{
${ }^{2}$ L'Institute for Development Studies, un think tank d'intellectuels vietnamiens, a été interdit en 2009 suite à sa prise de position trop virulente sur les politiques de l'Etat.

3 «Ethnique » désigne des populations linguistiquement et culturellement spécifiques et distinctes de l'ethnie démographiquement majoritaire à l'échelle nationale (les Kinh).
} 
la population ne court pas après les projets, ce sont les projets qui courent après la population.

Il convient de préciser que notre position sur le développement est influencée à la fois par notre expérience des processus de développement volontariste au Vietnam et par les analyses d'auteurs tels que R. Jaulin (1999), G. Rist (2001) et S. Latouche (2003) pour qui les fondements du développement s'inscrivent dans la lignée de la logique coloniale, de l'évolutionnisme social et du mythe du progrès et de la modernité. Ainsi, les risques de déséquilibres provoqués par les projets nous incitent plutôt à ne pas y participer et à les critiquer.

Cependant, témoins de nombreuses actions de développement que nous considérions néfastes pour les bénéficiaires, et régulièrement sollicités par les développeurs ou les pouvoirs publics pour les aider dans leurs projets, nous n'avons pas pu rester neutres et distants. Plutôt que d'observer sans intervenir, il nous a semblé nécessaire de nous engager. Notre intervention reste néanmoins marquée par notre position idéologique anti-développementaliste. Ainsi, plutôt que d'initier des changements, l'objectif était surtout de limiter les impacts négatifs d'actions exogènes. L'une des difficultés a alors été de ne pas projeter sur les populations locales notre propre vision, surtout dans ce contexte où la plupart des villageois adhèrent à l'idée que la " modernisation " (hiện đại hóa) est inéluctable, nécessaire et avantageuse, même si leurs conceptions de celle-ci sont plurielles et ne correspondent pas exactement à la vision techniciste dominante en Occident.

De notre point de vue, il était plus efficace de construire une action de développement à partir d'enquêtes qualitatives longues, sans prédéfinir ni les besoins des populations, ni le secteur $d^{\prime}$ 'intervention, ni les manières de mener l'action. Autrement dit, nous voulions montrer qu'il est préférable de ne rien prévoir quant aux actions concrètes à effectuer, et de mener en premier lieu une recherche approfondie et transversale au bout de laquelle un éventuel projet de développement serait écrit puis mis en œuvre. 
Afin de vérifier l'intérêt d'une telle démarche, nous avons créé en 2009 le " projet d'étude socio-anthropologique du changement social et du développement dans les villages ethniques au nord du Vietnam ". II s'agissait de mener des enquêtes qualitatives de type anthropologique dans une localité, afin d'identifier un éventuel secteur d'intervention et une manière appropriée de mener l'opération. Les résultats de cette étude qui portait autant sur les dynamiques sociales et culturelles que sur l'histoire locale du développement, nous ont conduits à endosser le rôle de médiateur entre les villageois et une action de développement conçue en dehors de la localité et potentiellement source de déséquilibres. Si le passage à l'acte (Dozon, 1991) était la condition pour confirmer le bien-fondé de notre posture, l'enjeu était de nous situer dans cet " entre-deux entre engagement et distance " (Atlani-Duault et Vidal, 2009 : 39).

Cet article présente comment nos positions se sont traduites concrètement dans nos pratiques de recherche et dans les interventions réalisées sur le terrain, qui témoignent en définitive d'une combinaison entre une recherche scientifique et un positionnement politique. Avant de présenter la manière dont ce projet a été mis en œuvre, nous allons rappeler les positions et les principes qui le fondent, puis les conditions dans lesquelles nous avons élaboré et mené notre étude, ainsi que les défis qu'il a fallu relever. La dernière partie sera consacrée à l'action de développement dans laquelle nous nous sommes impliqués.

Des constats et des principes à l'origine d'une position engagée

Ce projet découle d'un ensemble de constats sur les problèmes récurrents que présentent les actions de développement et qu'il s'agissait avant tout de comprendre pour ne pas les reproduire. 


\section{Chercher des problèmes aux solutions}

Dans la majorité des projets, les problèmes à résoudre et les secteurs d'intervention sont souvent prédéfinis en fonction des appels d'offres, des domaines de prédilection des opérateurs qui répondent aux appels (le genre, l'eau, l'environnement) et, plus généralement, des préoccupations et des idéologies des bailleurs et des grandes institutions internationales (le développement des infrastructures, la "bonne gouvernance ", les Objectifs du millénaire, le changement climatique, etc.).

Il en résulte que les domaines d'intervention, identifiés au préalable sans enquête rigoureuse, ne correspondent pas nécessairement aux conditions locales ni aux aspirations effectives des populations. Lors de l'élaboration et de la mise en œuvre des projets sur le terrain, les praticiens du développement s'évertuent alors à traiter des problèmes découlant de "solutions " définies $a$ priori et hors contexte (Naudet, 1999).

\section{Plier la réalité locale aux termes de référence (TDR)}

Tous les projets sont fortement contraints par des "termes de référence" (TDR) et des "cadres logiques", souvent rigides et conçus trop loin des réalités des populations ciblées, mais qui ont force de contrat avec les bailleurs de fonds (Giovalucchi et Olivier de Sardan, 2009). Les opérateurs locaux n'ont donc pas d'autre choix que de plier la réalité à ces TDR ou, quand ce n'est pas possible, d'" habiller " les rapports pour correspondre aux attentes et aux plans du projet fixés au préalable.

\section{Un usage périphérique des sciences sociales}

La temporalité des projets n'accorde généralement que peu de temps aux études de la situation locale. Quand les sciences sociales ont une place, leur rôle reste globalement périphérique (Guichaoua et Majeres, 1982 ; Lavigne Delville, 1997). Les chercheurs sont généralement appelés soit pour effectuer en peu de temps des études préalables, consistant à évaluer des besoins sur des aspects confinés de la réalité locale; soit en tant que "pompiers de 
service " (Lavigne Delville, $1997:$ 7), pour résoudre des problèmes dans le déroulement du projet, généralement considérés de l'ordre des " blocages culturels ». Mais, dans tous les cas, ils sont sollicités alors que les objectifs et les secteurs sont déjà prévus si bien que, sauf exception ${ }^{4}$, il n'est pas possible de changer les structures du projet (logiques, objectifs, orientations) et encore moins d'abandonner ce dernier si les résultats des enquêtes le préconisent.

Enfin, s'il est aujourd'hui admis que la prise en compte des facteurs culturels et sociologiques est nécessaire, il est en revanche plus rare de considérer que la "configuration développementaliste ${ }^{5}$ soit aussi un objet d'étude. Or, le fonctionnement d'un projet est autant tributaire de ceux qui le reçoivent que de ceux qui l'administrent.

\section{Faible connaissance de la réalité locale}

Ces constats indiquent qu'il subsiste une distance souvent considérable entre les réalités locales - complexes, articulées, évolutives, parfois instables - et les projets, leurs objectifs, leurs logiques, leurs contraintes et leurs solutions prédéfinies. Cette inadéquation limite la réussite des projets, voire endommage les équilibres politiques, environnementaux ou socio-économiques en place. Si les échecs des projets sont parfois avoués, les éventuels " dégâts " provoqués dans leur sillage sont rarement évoqués et, s'ils le sont, ils sont rarement rendus publics (Arditi, 2005 ; Aubriot, 2015).

\footnotetext{
${ }^{4}$ Lavigne Delville donne « un exemple - rare ! - où une étude de faisabilité débouche sur un abandon du projet, et qui plus est pour des "raisons sociologiques" » (1997 : 25).

${ }^{5}$ Elle renvoie à " cet univers largement composite de responsables d'ONG, de chercheurs, de techniciens, de chefs de projets, d'agents de terrain, qui vivent en quelque sorte du développement des autres, et mobilisent ou gèrent à cet effet des ressources matérielles et symboliques considérables » (Olivier de Sardan, 1995 : 7).
} 
En réaction à ces constats, nous avons défini une série de principes, qui sont à la base de notre projet et qui ont conditionné notre intervention :

Principe d'Hippocrate: "D'abord, ne pas nuire " (Anderson, 1999), étant entendu que l'évaluation du " nuire » peut varier beaucoup selon les perspectives et les idéologies de chacun. Pour nous, cela signifiait non seulement ne pas affecter négativement les conditions de vie matérielles et les conditions environnementales, mais aussi ne pas altérer la culture et l'organisation sociale en place.

- Principe de connaissance préalable: aucun changement social impulsé de l'extérieur n'est approprié ni durable sans une connaissance précise et préalable des réalités locales sur lesquelles il va s'appuyer. Il convient donc de se donner les moyens de comprendre les réalités locales avant de tenter de les transformer (Olivier de Sardan, 1995). Et, concernant l'appréhension des situations sociopolitiques locales, il est impératif de mobiliser les savoirs et méthodes de la recherche en sciences sociales et non pas uniquement des expertises dans le cadre d'enquêtes rapides (de types RRA et PRA6).

- Principe d'interdépendance du social : au sein d'un groupe social, les différents domaines de l'existence sont interconnectés et interdépendants. Le projet doit donc prendre en compte cette globalité, et les actions menées doivent être considérées comme des éléments supplémentaires venant s'ajouter à un équilibre social déjà existant (Jaulin, 1999).

- Principe de prudence : une démarche «attentive à la singularité des cas, qui est capacité à délibérer et à décider dans une situation d'incertitude, marquée par la

\footnotetext{
${ }^{6}$ RRA : Rapid Rural Apraisal ; PRA : Participatory Rural Apraisal.
} 
contingence " (Larrère et Larrère, $1997: 246)$. Cette prise en compte des circonstances spécifiques et de leur imprévisibilité implique de mener une étude et une intervention micro-localisées, qui excluent toute tentative de changement à un niveau macro.

Ces principes, déjà identifiés par les anthropologues du développement et parfois intégrés par les développeurs, restent difficiles à mettre en œuvre lors du passage à l'action compte tenu des diverses contraintes temporelles, budgétaires, méthodologiques, techniques et idéologiques inhérentes aux projets de développement. Nous avons néanmoins tenté de les appliquer à travers un projet dont l'objectif consistait à étudier en profondeur la situation locale avant de concevoir et de mener une action de changement. L'idée sous-jacente n'était pas de monter un projet de développement mais de prouver la pertinence d'une démarche.

\section{Comprendre comment le monde se transforme}

\section{Le montage du projet : défis, contraintes et adaptations}

Un défi majeur était de trouver des financements afin de mettre en pratique les positions présentées ci-dessus. Nous avons alors rédigé un premier projet qui comportait explicitement deux composantes : un volet recherche et un volet action.

Le volet recherche s'articulait autour de deux axes principaux. Le premier était une étude des dynamiques sociales, culturelles, politiques et économiques. Le second consistait à étudier les différentes facettes des processus de développement et de changement social propres à la commune d'étude. En s'appuyant sur les résultats de ces recherches transversales, le second volet concernait la conception, la réalisation et le suivi d'un projet de développement spécifique, construit sur mesure.

La première difficulté a été de convaincre des bailleurs pour un projet qui proposait avant tout une démarche et de la recherche et 
non un plan d'action concret et détaillé. Après quelques échecs auprès d'ONG et suite à de longues et passionnées discussions avec le directeur (de l'époque) de l'AFD au Vietnam, le département recherche de I'AFD a accepté de nous financer pour deux années ${ }^{7}$. Nous avons bénéficié d'une conjoncture très favorable puisque notre interlocuteur, que nous ne connaissions pas mais avec qui des affinités intellectuelles se sont rapidement nouées, était sociologue et défendait vigoureusement l'usage des sciences sociales dans les projets de développement. II s'est donc révélé particulièrement sensible à notre initiative. En outre, récemment arrivé au Vietnam, dans un contexte où le département recherche de I'AFD était particulièrement disposé au financement de nouveaux projets, il a pu débloquer des fonds pour de petits projets expérimentaux, habituellement non soutenus par cette institution. Ce partenariat avec un pôle recherche d'une agence de développement s'avérait pertinent puisqu'il reflétait les deux composantes de notre projet. De plus, le bailleur ne nous a pas imposé de directives particulières, si ce n'est d'ajouter au document final du projet des orientations pour la lutte contre la pauvreté. Notre intervention ainsi que la problématique n'étaient pas commanditées par des développeurs, c'était une demande de notre part. La " coopération négociée " entre les chercheurs et les agences de développement (Olivier de Sardan, 1995 : 194) a donc été à la base du montage de ce projet.

La seconde difficulté a été de trouver un organisme d'Etat vietnamien prêt à se porter garant pour nous. En effet, au Vietnam, toute enquête de terrain est conditionnée par des autorisations officielles précisant les lieux, les dates, les personnes rencontrées et les thèmes d'enquête abordés. Depuis 1998, nous avions des relations de coopération, d'échanges et d'obligations réciproques avec le département de la culture, du sport et du tourisme de la province de Lào Cai (DCLC), et en particulier avec son directeur. Ce département a ainsi accepté de devenir notre partenaire officiel.

7 Suite au changement de partenaire vietnamien, le projet durera une année supplémentaire, soit un total de trois ans sur un financement de deux ans. 


\section{Le déroulement de l'enquête}

Une fois le montage financier et institutionnel mis en place, nous avons commencé les recherches dans une commune rurale du nord du Vietnam que nous avons choisie en fonction d'une série de critères: une commune peuplée de groupes ethniques des montagnes considérés comme relativement pauvres, une localité géographiquement et administrativement accessible mais à distance des organes de décision centraux, un espace restreint constituant une société d'interconnaissance directe ouverte sur l'extérieur.

La commune d'étude est située au sud-est de la province de Lào Cai. Cette province est à l'extrême nord du Vietnam, frontalière avec la Chine. La commune est composée de quatre groupes ethniques : les Tày (95\%), les Hmong, les Dao et les Kinh (5\%), soit 4800 habitants, répartis dans 1012 familles $^{8}$. La principale activité est la riziculture irriguée en fond de vallée (altitude $200 \mathrm{~m}$ ) associée à de l'élevage et quelques emplois extra-agricoles (commerçants, manœuvres, maçons, etc.). La limite du terroir s'étend sur les crêtes des montagnes à plus de $1000 \mathrm{~m}$ d'altitude. La plupart des terres de collines sont occupées par des cultures annuelles (manioc et maïs), des plantations d'arbres à croissance rapide (acacia, styrax) et par plusieurs dizaines d'hectares de thé, reliquat de projets d'Etat du début des années 2000 .

Nous avons opté pour une approche classique de l'anthropologie. Notre étude était micro-localisée, ciblée sur un terrain d'étude spatialement et socialement circonscrit. Cette petite unité d'espace et d'organisation sociale a facilité notre appréhension des relations sociales et politiques nouées autour du changement social et des projets de développement. Au niveau des chercheurs, le noyau dur de notre équipe était composé de trois fonctionnaires vietnamiens du DCLC (deux sociologues et une anthropologue) et de deux anthropologues français (un chargé de

\footnotetext{
${ }^{8}$ Statistiques communales de 2012.
} 
recherche du CNRS et un jeune docteur spécialiste du Vietnam rural). Tous les chercheurs avaient au moins cinq ans d'expérience d'enquête en milieu rural et ethnique au Vietnam. Ils maîtrisaient donc la langue vietnamienne, qui est couramment parlée par la population locale, bien qu' elle ne soit pas sa langue maternelle.

L'immersion et les entretiens approfondis ont été nos principales techniques d'enquête. II s'agissait de conduire des entretiens ouverts tout en suivant le rythme local, en participant aussi bien aux activités agricoles variées (plantation du riz, récolte du thé et du manioc, coupe de bois...) qu'aux multiples activités sociales (banquets de mariage, de funérailles et d'inauguration de nouvelles maisons), sans oublier les invitations du comité populaire ${ }^{9}$ pour assister aux réunions de lancement de nouveaux projets de développement. Les chercheurs de l'équipe étaient basés dans des villages différents ${ }^{10}$ et logeaient chez les villageois. Nous nous étions réparti des thèmes de recherche, tout en collectant des informations sur les thèmes des autres. Nous organisions des réunions régulières pour recouper nos informations et analyser les données. Pour compléter nos connaissances sur une activité importante des villageois mais au sujet de laquelle nos compétences étaient limitées, nous avons impliqué, en tant que stagiaire, une étudiante française en agronomie afin d'établir un diagnostic des systèmes de production agricole.

Disposant de plusieurs mois d'enquête par an, nous avons pu alterner les interviews ciblées sur nos thèmes de recherche avec de nombreuses heures de discussions ouvertes avec les paysans et les responsables politiques, sur les thèmes qui leur tenaient particulièrement à cœur, comme la rupture des canaux d'irrigation construits l'année précédente, les raisons de la chute du prix du manioc, les retards d'indemnisation pour les propriétaires de terres expropriés, les tensions sur les espaces de pâturage, etc. En trois ans d'étude (2010-2013), totalisant plus de 140 jours d'enquête de

\footnotetext{
${ }^{9}$ Equivalent de la mairie de commune.

${ }^{10}$ La commune comprend seize villages au total.
} 
terrain par chercheur, notre équipe a ainsi interrogé plus de 400 personnes, paysans autant que responsables locaux (de la commune et du district $)^{11}$. Nous avons aussi participé à plus d'une douzaine de réunions officielles avec les autorités de la province et du district.

\section{Les thèmes de recherche}

Sur le plan de la recherche, le premier axe a consisté en une étude anthropologique transversale de la commune. Notre approche a été volontairement non sectorielle avec pour objectif d'obtenir une vision globale, quoique non exhaustive, des activités quotidiennes des villageois. Nous avons par exemple autant tenté de reconstituer les évolutions historiques locales, de l'époque précoloniale à aujourd'hui, que de cerner les pratiques de sociabilité, les cérémonies et les rituels. Nous avons également étudié les activités économiques agricoles et extra-agricoles, les principales sources de revenus monétaires, les formes d'accès au crédit mais aussi la circulation non marchande (entraide, dons et contre-dons), les enjeux fonciers et l'organisation du pouvoir local. Si nous n'avons pas pu documenter avec le même degré de précision tous les thèmes de recherche, ils figuraient tous dans nos canevas d'entretien.

Le deuxième axe de la recherche a porté sur les dynamiques de changement social ainsi que les projets de développement passés et en cours, leurs configurations, leurs logiques et les réactions locales. Dans la commune d'étude, le nombre de projets de développement mis en œuvre est considérable, surtout si on inclut les transformations impulsées par le pouvoir central dans le sillage de la Révolution à partir de 1954 (réforme agraire, coopératives agricoles, écoles, centres de santé, semences améliorées), localement perçues dans la même logique que les projets de développement actuels. Ainsi, pour les villageois et les responsables locaux, les projets de développement participent à la construction de l'histoire locale, ils façonnent le paysage physique (défrichage,

\footnotetext{
${ }^{11}$ Ces derniers sont souvent également paysans.
} 
irrigation, infrastructure, etc.) autant que le paysage socioéconomique. Ils déterminent aussi, mais différemment selon les acteurs considérés, le fonctionnement des nouveaux projets. Une connaissance approfondie de la manière dont les activités des projets précédents avaient été menées, puis reçues et transformées par les populations locales et les autorités communales, nous semblait donc essentielle avant de prétendre initier un nouveau projet.

Dans un premier temps, nous avons dressé le panorama de l'ensemble des projets de développement appliqués à la commune depuis quinze ans : plus de vingt projets ont été recensés, touchant une grande quantité d'activités agricoles, économiques, sociales et culturelles. Ensuite, en plus des deux plus grands programmes gouvernementaux multi-activités qui concernent les infrastructures locales (routes, écoles, marchés, électricité, hydraulique, etc.) et l'économie domestique ${ }^{12}$, nous avons ciblé quelques projets significatifs. La démarche consistait à étudier les relations entre les acteurs impliqués puis à mettre en perspective les différents intérêts, logiques et normes qui se rencontrent, et parfois s'affrontent, au cours des projets. Nous avons ainsi étudié en détail une série de projets.

Trois projets de mise en place d'un réseau "d'eau propre " se sont succédé depuis 2002, visant à donner un accès stable mais payant à l'eau de consommation pour les ménages. Dans la majorité des cas, les villageois ont combiné leur système local gratuit d'adduction d'eau avec le nouveau système, payant, utilisé uniquement lors de besoins importants en eau (cérémonies, fêtes) ou de manques temporaires. Certains villages, dont les besoins en eau étaient déjà largement satisfaits, ont refusé le nouveau système, provoquant des tensions avec les autorités locales chargées du projet.

12 Programm for Socio-economic Development in Communes faced with Extreme Difficulties - dit P135 (1998-2015) - et le programme "Construire une nouvelle campagne » (2010-2020). 
Deux projets de plantation et de commercialisation de thé ont été lancés en 2001. L'Etat a subventionné les plants de thé et a garanti la vente de la production via des contrats entre des compagnies privées et les paysans. Mais, après deux ans, les compagnies ont arrêté de s'approvisionner dans la commune. Les paysans se retrouvant avec du thé invendu ont finalement remplacé cette production par celle du manioc, moins rentable mais offrant des revenus plus réguliers.

Des formations nécessaires pour candidater aux postes de fonctionnaires ont été organisées dans la commune. Théoriquement ouvertes à toute personne ayant le niveau requis, elles ont été monopolisées par les agents déjà en place au profit de leur famille. Les personnes n'appartenant pas au «cercle des fonctionnaires » ont été écartées de l'accès à la fonction publique.

Le programme "Construire une nouvelle campagne » a financé un projet de diffusion de semences de riz japonais de haute qualité. Les semences de riz n'étaient pas adaptées aux conditions agroécologiques locales, et les soins nécessaires étaient peu compatibles avec les compétences et les techniques des cultivateurs. La récolte du premier cycle s'est donc soldée par un échec majeur avec la perte de plus de $50 \%$ de la production. Les familles impliquées, n'ayant plus de riz pour leur propre consommation et pas de riz à vendre, se sont retrouvées dans une situation de pénurie. Les autorités locales, soucieuses de ne pas être en contradiction avec les directives des instances supérieures et de ne pas être mises en cause dans les raisons de l'échec, n'ont pas fait état de la situation dans leurs rapports, si bien qu'un deuxième cycle a été planifié par la province. Afin de ne pas mettre à nouveau les villageois - qui sont aussi leurs voisins, leurs amis et leurs parents - dans une situation critique, les autorités ont planté ces semences subventionnées sur leurs propres parcelles. Cet exemple est révélateur de I'inadéquation de certains projets par rapport aux réalités locales. II témoigne aussi des enjeux liés à la double appartenance des cadres communaux, à la fois responsables politiques et membres de la communauté, qui jouent parfois le rôle de «tampon ». 
Le programme national d' "Edification d'un mode de vie civilisé " a été mis en œuvre dans la commune d'étude à partir de 2002. L'objectif était d'“ aider" les ethnies dites minoritaires à se " moderniser » et à s'intégrer à la nation. Cette campagne visait à réformer un large éventail de pratiques et de coutumes jugées primitives ou "féodales». Les villageois ont déployé diverses stratégies d'accommodement, entre acceptation, assimilation, évitement et résistance passive. Ces réactions sont significatives de la manière dont les populations locales intègrent et transforment les interventions extérieures.

Enfin, un projet de planification urbaine du centre de la commune (2010-2015) avait pour objectif de créer le troisième centre urbain du district. Ce projet, qui prévoyait la construction de rues goudronnées avec un tout-à-l'égout, d'un grand marché et de maisons en briques et ciment, impliquait l'expropriation et le déplacement de quelques foyers. Certains cadres de la commune, informés du projet et liés entre eux par des relations de parenté et d'alliance, ont acheté l'essentiel des terrains les mieux situés (au bord des routes principales et autour du marché) avant l'annonce officielle du projet. Ils ont ainsi bénéficié de l'augmentation du prix du foncier et des opportunités commerciales engendrées par la restructuration du centre de la commune.

\section{Le passage à l'acte}

En dehors de connaissances académiques sur les réalités sociales des groupes ethniques des régions montagneuses du nord du Vietnam, les résultats de nos recherches ont permis d'établir un diagnostic de la situation locale et d'évaluer les possibilités d'initier une action de développement. Nous ne détaillons pas ici les résultats des enquêtes. Seules les conclusions transversales qui nous ont permis de réaliser le diagnostic et de définir la manière la plus appropriée d'agir sont présentées. 


\section{Diagnostic sur la situation locale}

Notre étude des dynamiques socio-économiques a montré que les besoins locaux tels qu'ils sont perçus par les habitants sont globalement satisfaits. En effet, les villageois n'expriment pas de manque majeur et ne réclament pas spontanément d'intervention extérieure pour améliorer leur quotidien. $\mathrm{Si}$, au regard des critères internationaux, beaucoup de foyers sont en dessous du seuil de pauvreté, au niveau local le taux de pauvreté est relativement faible par rapport à d'autres communes. II était de $16 \%$ en 2008 , alors que le niveau moyen pour la province était de $20 \%$. D'autres formes de pauvreté économique et un manque structurel de revenus monétaires sont observables chez certains foyers, mais la capacité des habitants à satisfaire une part importante de leurs besoins de base (alimentation, logement, lutte contre le froid, accès à l'eau, accès aux services de santé, etc.), associée aux obligations sociales d'entraide, empêche les plus démunis de sombrer dans la misère.

En outre, notre étude du changement social sur le long terme et des projets de développement passés a indiqué que l'Etat prenait déjà en charge un grand nombre d'actions de développement local, notamment dans le domaine des infrastructures, de la santé, de l'eau, de la formation et de l'éducation, du développement agricole et forestier, et de l'accès au microcrédit. Cette situation ne signifiait pas que tous ces domaines ne comportaient pas de difficultés pour les habitants, mais elle indiquait qu'aucune action n'était envisageable en dehors des réseaux et des logiques de l'Etat. II y a en effet un encadrement étroit et fort de toute action extérieure par les autorités politiques, ce qui limite la marge de manœuvre et l'indépendance vis-à-vis des logiques bureaucratiques, productivistes et évolutionnistes qui guident les processus de développement au Vietnam.

En résumé, notre premier constat a été le suivant : il n'était pas pertinent de lancer un projet supplémentaire dans cette commune. 
Les problèmes et les déséquilibres rencontrés localement ${ }^{13}$, et qui pouvaient justifier une intervention, provenaient en fait pour la plupart d'influences extérieures généralement à caractère topdown, non adaptées aux réalités locales, encadrées par l'Etat ou liées à l'intégration et à la dépendance accrue au marché.

\section{Intervenir comment ? La position de médiateur}

II a résulté de cette analyse que la seule action pertinente était celle de médiateur ou d'intermédiaire entre ce qui venait de l'extérieur et les populations locales (Dozon, 1991 ; Atlani-Duault, 2007). La plupart des interventions étant orchestrées ou encadrées par l'Etat, c'était avant tout en agissant auprès des décideurs politiques aux différents niveaux de l'appareil bureaucratique qu'il serait possible d'influer sur les conditions des changements instaurés localement, notamment en essayant d'agir en amont, c'est-à-dire dès la conception des projets. Autrement dit, plutôt que de concevoir un projet de développement additionnel, il semblait plus pertinent et efficace de s'appuyer sur ceux en cours ou à venir pour les orienter et favoriser leur meilleure adéquation avec les réalités locales, en vue de limiter autant que possible les risques de dysfonctionnements qu'introduisent des interventions extérieures. Notre étude des réactions locales face aux projets passés nous permettait également de confirmer que toute action devait présenter une flexibilité permettant des accommodements et des adaptations par les populations locales. La structure même des projets devait ainsi prévoir une marge de manœuvre facilitant les réappropriations locales.

Notre diagnostic étant posé, la question restait de savoir si nous allions assumer nos conclusions et essayer de mettre en œuvre les formes d'intervention préconisées. La posture de lobbying que nous recommandions en définitive nous apparaissait peu attrayante dans

\footnotetext{
${ }^{13}$ Manque de ressources monétaires, surendettement, inégalités sociales et faible possibilité de mobilité sociale, possibilités limitées de revendication politique libre, problèmes de pollution et de gestion des déchets, érosion et appauvrissement des sols, mesures ethnocidaires sur les cultes et les rituels, etc.
} 
le contexte vietnamien : risquée, illégitime et surtout hors de notre ressort. Mais, finalement, les circonstances nous ont poussés à revoir notre position.

\section{Intervenir sur quoi ? Accompagner et orienter un projet de développement touristique}

Comme nous l'avons évoqué plus haut, un programme de " développement urbain » se mettait en place dans le centre de la commune. En parallèle, nous avions entendu parler d'un plan provincial pour ouvrir l'ensemble de la zone au développement touristique. L'un comme l'autre étaient censés stimuler la croissance économique et le développement local mais ils étaient contradictoires dans leurs objectifs ${ }^{14}$ et comportaient le risque d'accroître les inégalités entre le centre et la périphérie de la commune.

Dans ces circonstances, notre équipe a été sollicitée par les responsables du district pour les conseiller dans la mise en place du projet touristique, qui n'était alors pas encore rédigé ni financé. Cette invitation était le fruit d'un dialogue constructif progressivement établi avec les autorités et le résultat d'échanges imprévisibles, publics et privés, entre les diverses parties prenantes. Elle était également liée au fait que l'un des membres français de l'équipe avait déjà été impliqué, en tant que consultant, dans un projet de développement touristique dans la même province (Culas, 2004). Cette invitation comportait pour nous le risque d'être complices d'un changement imposé de l'extérieur, possiblement déstructurant au niveau local. Mais elle constituait aussi une opportunité inédite de se greffer sur une dynamique de développement dès sa conception pour favoriser son adéquation avec les réalités locales et en limiter ainsi les effets possiblement négatifs. Autrement dit, nous étions face à l'opportunité de mettre

\footnotetext{
${ }^{14}$ D'un côté " urbaniser » signifie remplacer les maisons traditionnelles sur pilotis en bois par des maisons " modernes » en briques et ciment; de l'autre "développer le tourisme ethnique " nécessite de conserver et entretenir ces maisons en bois, attractives pour les touristes.
} 
en œuvre les recommandations prônées par notre étude. Encore fallait-il être en mesure de pouvoir influencer les orientations du projet et, par exemple, de convaincre les autorités qu'il était préférable de faire un projet d'écotourisme responsable de petite échelle au lieu d'envisager, comme c'était le cas, un projet de tourisme de masse avec hôtels, karaoké et "folklorisation " de la culture locale. En effet, comme cela a été souligné plus haut, les conditions de notre participation à une intervention de développement étaient fixées par une série de principes qu'il s'agissait alors de ne pas perdre de vue, notamment celui préconisant: " avant tout ne pas nuire ». Pour nous, I'ambition immédiate du projet était de limiter les impacts potentiellement négatifs du tourisme (Dolezal, 2011) tout en tempérant les déséquilibres et les inégalités que le développement urbain du centre de la commune allait par ailleurs engendrer; l'idée de générer des sources de revenus d'appoint ne venait qu'en second lieu.

Notre relation personnelle et professionnelle avec le directeur du DCLC a également influencé notre choix de participer à ce projet. En effet, devant l'important travail de lobbying nécessaire pour réorienter les objectifs initialement prévus et insérer nos principes, il était impératif d'avoir les moyens d'influencer ce décideur haut placé, qui était celui qui validerait le lancement du projet. Enfin, les relations d'écoute mutuelle et de collaboration sincère avec certains des cadres du district nous ont également incités à « tenter le coup ".

Ainsi, suite à plusieurs réunions et quelques banquets festifs avec les autorités du district, où nous explicitions les risques du tourisme et les contradictions entre le développement urbain et le tourisme "ethnique ", et grâce à un travail de fond avec le directeur du DCLC, nous avons réussi sans trop de difficultés à nous mettre d'accord sur la mise en place d'un projet pilote d'écotourisme de petite envergure. De manière plus implicite, l'enjeu était de réduire les transformations subies par les bénéficiaires et de modifier l'ensemble du projet pour qu'il soit plus adapté et adaptable à ceux-ci. D'autant qu'à ce moment, les 
populations locales n'avaient pas encore été consultées ni informées. Notre équipe a ainsi signé un accord de collaboration avec le comité populaire du district pour l'assister dans la conception, la réalisation et le suivi de ce projet touristique. En marge des réunions officielles, où il s'agissait surtout de poser et négocier les bases idéologiques du projet à la jonction des intérêts des différents acteurs, nous avons organisé des groupes de travail restreints avec la personne responsable de la rédaction du projet et avec le vice-président du comité populaire du district en charge de soumettre le projet aux échelons supérieurs. Notre étude des projets passés et des processus de prise de décision nous avait en effet appris à être attentifs à ces différents modes d'échanges, où le formel et l'informel s'entremêlent.

Si nos enquêtes passées dans la commune nous donnaient des pistes sur la manière de mettre en œuvre un projet, elles ne nous indiquaient pas comment concevoir spécifiquement un projet de tourisme. En parallèle à ce travail politique et relationnel, nous avons donc sollicité différents experts du tourisme au Vietnam et visité, avec nos principes en ligne de mire, différents sites écotouristiques afin d'identifier les biais et les points forts de chaque projet. Sur la base de ces informations, nous avons ainsi mis sur papier les orientations majeures : plutôt que de bâtir des hôtels et des restaurants au centre de la commune, le logement chez l'habitant dans les villages périphériques serait privilégié, puisque plus de $80 \%$ de l'habitat est constitué de maisons traditionnelles sur pilotis. Afin de limiter les perturbations liées à l'arrivée de touristes, il n'était pas prévu que les nouvelles activités liées au tourisme remplacent les autres activités. Ce choix a découlé de nos enquêtes passées qui montraient que, lorsque les villageois exerçaient une nouvelle activité génératrice de revenus, ils n'abandonnaient par leurs activités agricoles, même s'ils occupaient une fonction de cadre communal ou de commerçant. Ce choix a également été conforté par le fait que le tourisme est une activité irrégulière et risquée, et que le nombre estimé de touristes ne permettrait pas aux familles d'en vivre toute l'année. 
Un objectif important, identifié lors de l'étude des autres projets touristiques de la région et dans la littérature spécialisée (Dolezal, 2011), était aussi de veiller à ce que ce projet touristique n'entraîne pas l'apparition d'inégalités nouvelles au niveau local. Sur ce point, nous avons préconisé la mise en place d'une caisse villageoise et d'un système de répartition des activités (logement des touristes, vente des produits locaux, fonctions de guide) entre les différents foyers du village.

En termes de gestion, le système serait fondé sur une coopération entre différents groupes d'acteurs : les populations, les autorités locales (commune, district et province), un comité local de gestion, une centrale de réservation à Hanoi, des agences de tourisme responsables et les chercheurs. L'idée était de créer des conditions pour que les changements soient négociés entre les diverses parties prenantes. La gestion du tourisme devrait ainsi à la fois être assurée par le comité local de gestion, composé des personnes impliquées dans le projet, de cadres officiels et de villageois détenant une autorité légitime localement établie (chefs de lignage), et par la centrale de réservation extérieure à la communauté. Cette dernière se chargerait de la liaison entre les villages et les touristes et assurerait les liens entre le comité local de gestion et les agences de voyages susceptibles d'envoyer des touristes dans la localité. Cette gestion à double niveau vise à limiter l'accaparement des bénéfices par les autorités ou par des personnes de pouvoir et à empêcher l'exploitation du tourisme par des agences touristiques peu soucieuses des équilibres locaux, tout en assurant un fonctionnement adapté aux rythmes locaux. Par exemple, le comité de gestion et la centrale de réservation seraient régulièrement informés des activités sociales, économiques et culturelles importantes ayant lieu dans la commune (récoltes, inauguration de nouvelles maisons, mariages, funérailles, cérémonies diverses, etc.) et répartiraient les touristes dans les villages en fonction des occupations des habitants.

A ce stade où, déjà, les grandes lignes du projet étaient tracées, I'un des enjeux principaux consistait à intégrer davantage le point de vue des villageois dans la conception et la mise en œuvre du 
projet. Pour cela, et afin de rester en accord avec nos principes, des enquêtes spécialement appliquées au projet de tourisme étaient nécessaires pour identifier les villages et les itinéraires touristiques, sélectionner les familles intéressées, établir le comité de gestion, organiser des réunions et des formations (hébergement, cuisine et guide local), etc.

Le dernier défi à relever a ainsi été de trouver des sources de financement pour ces activités et pour acheter le matériel nécessaire (matelas, moustiquaires, W.-C., douches, vélos, etc.). Car, si le développement du tourisme était programmé par les autorités provinciales, aucuns fonds n'avaient pour le moment été mis à disposition.

Ayant changé ses objectifs, l'AFD n'était plus prête à nous financer. Nous avons tenté une collaboration avec une ONG de petite taille, et donc très flexible, dont les activités n'étaient pas orientées vers la seule génération de revenus monétaires. La difficulté de convaincre les bailleurs internationaux pour ce projet qui ne correspondait pas aux standards attendus et dont les retombées économiques s'avéraient faibles, nous a finalement écartés des sources de financements internationaux et de I'ONG. Mais, comme la perspective d'ouvrir la zone au tourisme était au départ une initiative des pouvoirs publics vietnamiens, des fonds ont pu être dégagés par ce biais.

En 2015, le projet a ainsi obtenu un financement par l'Association de lettres et arts folkloriques du Vietnam, dont le vicedirecteur est l'ancien directeur du DCLC, avec qui nous étions liés depuis le début du projet. Ces financements ont permis de lancer les activités de préparation.

Nous sommes actuellement en train de mener ces actions. L'enjeu central est d'impliquer les villageois dans le projet qui a été envisagé de manière suffisamment flexible pour pouvoir en changer les structures. A titre d'exemple, nous rencontrons actuellement les habitants des villages sélectionnés, chez eux, pour leur expliquer personnellement le projet, leur demander leur avis, voir ceux qui souhaitent et peuvent participer, sachant que certains refusent 
faute de temps et de main d'œuvre alors que d'autres s'emballent sans anticiper les répercussions concrètes. II s'agira ensuite d'organiser une réunion avec tous les villageois intéressés, afin qu'ils sélectionnent les quelques foyers qui commenceront à participer directement à l'activité touristique. Au regard des enseignements tirés de l'étude des projets passés, les réunions organisées dans la commune avec les habitants ne s'effectuent pas uniquement dans le formalisme du comité populaire de la commune, mais consistent à inviter les intéressés à participer à un repas festif chez l'un d'entre eux et à discuter du projet avant le repas, mais aussi pendant et après, quand la convivialité permet de s'exprimer plus librement que dans les salles des bâtiments administratifs.

Entre 2014 et 2015, quatre maisonnées ont déjà accueilli une cinquantaine de touristes, à titre expérimental. Nous travaillons sur les feed-back des paysans, des touristes et des agences touristiques pour ajuster le projet.

\section{Conclusion}

Face aux constats sur les biais récurrents du développement, nous avons établi une série de principes qui définissent les conditions de notre implication dans des initiatives de changement social planifié. Nous avons ensuite tenté de les mettre en œuvre dans un projet de recherche appliqué à une situation de développement au Vietnam. Ce projet est né d'un pari. Nous voulions montrer qu'il est possible, voire préférable, de choisir le secteur d'intervention et de concevoir un projet de développement seulement après l'étude approfondie des réalités locales, et d'assurer ensuite le suivi du projet, en incluant une étude des développeurs et des développés. Les actions fondées sur cette démarche sont censées favoriser la réussite du projet en termes d'appropriation locale et d'amélioration durable des conditions de vie, tout en permettant de limiter les déséquilibres potentiels induits par un changement initié de l'extérieur. 
L'idée est donc de faire de la recherche anthropologique le point de départ d'un projet de développement, différemment de la position plus courante dans laquelle la recherche informe les acteurs d'un projet prédéfini ou les conseille sur une action en cours. Dans ce cas particulier, le mode d'intervention le plus pertinent a été de se situer en médiateurs entre les logiques locales et des interventions extérieures qui ne peuvent être évitées. Nous avons saisi la rare opportunité d'intervenir dans un nouveau projet dont nous avions la possibilité de transformer les objectifs et les actions afin d'en limiter les impacts négatifs. Nous sommes ainsi passés d'observateurs distants, à conseillers impliqués dans un projet de tourisme en construction, pour finir acteurs directs et observateurs d'un projet écotouristique.

Ce projet d'écotourisme n'est pas particulièrement innovant dans sa forme. Mais la manière d'intervenir dans ce domaine (à partir d'enquêtes socio-anthropologiques, sans secteur prédéfini), ses objectifs (réorienter un projet vers ce qui nous semblait le moins risqué en termes de déséquilibres locaux), puis la manière dont il a été conçu et mené (par un travail de lobbying auprès des décideurs associé à des enquêtes auprès de la population locale) sont, dans le contexte vietnamien, assez inédits.

Si les premières étapes de notre intervention sont réussies dans le sens où nous avons pu nous investir dans le projet dès sa conception, en changer les objectifs initiaux et introduire des éléments de la réalité locale, il reste encore à assurer le suivi et le développement des activités sans déroger à nos principes. Mais, dès à présent, cette expérience métisse entre anthropologie et développement devrait permettre d'apporter des éléments nouveaux et, peut-être, de nouvelles bases de dialogue pour une collaboration entre développeurs et anthropologues. 


\section{Bibliographie}

ANDERSON M.B. (éd.), 1999, Do No Harm: How Aid Can Support Peace - Or War, Boulder, Co. Lynne Rienner.

ATLANI-DUAULT L. (éd.), 2007, Humanitaire, 4, numéro spécial: Anthropologues et ONG : des liaisons fructueuses?

ATLANI-DUAULT L. et Vidal L. (éds.), 2009, Anthropologie de l'aide humanitaire et du développement, Paris, Armand Colin.

ARDITI C., 2005, « Niger : chronique d'une évaluation censure ou comment une agence des Nations Unies fabrique un bilan positif de son action contre l'insécurité alimentaire, la marginalisation des femmes et la pauvreté ", Revue Tiers Monde, 184 : 861-883.

AUBRIOT J., 2015, "Lever le tabou de l'échec pour mieux répondre aux enjeux de développement ", [en ligne], consulté le 06 novembre 2015, http://ideas4development.org/lever-le-tabou-de-lechecpour-mieux-repondre-aux-enjeux-de-developpement

BOUJU J., 2011, « Une application de l'anthropologie au développement. Le métier de praticien ", Cahiers d'études africaines, 202-203 : 563589.

CERNEA M., 1999, La dimension humaine dans les projets de développement: Les variables sociologiques et culturelles, Paris, Karthala.

Cahiers d'études africaines, 2011, Les sciences sociales au miroir $d u$ développement, 2-3/202-203, Paris, Edition de l'EHESS.

CULAS C., 2004, " Recommandations pour la préservation des futurs sites touristiques dans la province de Lào Cai ", Bordeaux, Coopération Région Aquitaine-Province de Lào Cai, multigr.

DOLEZAL C., 2011, “Community-Based Tourism in Thailand: (Dis-)Illusions of Authenticity and the Necessity for Dynamic Concepts of Culture and Power", Austrian Journal of South-East Asian Studies, 4(1) : 129-138.

DOZON J.-P., 1991, « Le dilemme connaissance/action : le développement comme champ politique ", Bulletin de l'APAD, 1 : 14-17. 
FONTANEL A., 2001, " Les fonctionnaires au Viêt Nam : une classe sociale en transition ", Raisons politiques, 3 : 55-65.

FFORDE A., 2009, Coping with Facts: A Skeptics Guide to the Problem of Development, Sterling, Kumarian Press.

GERONIMI V., BELLIER I., GABAS J.-J., VERNIERES M. et VILTARD Y. (éds), 2007, Savoirs et politiques de développement. Questions en débat à I'aube du XXIe siècle, Paris, Karthala/GEMDEV

GIOVALUCCHI F. et OLIVIER DE SARDAN J.-P., 2009, " Planification, gestion et politique dans l'aide au développement : le cadre logique, outil et miroir des développeurs ", Revue Tiers Monde, 198 : 383-406.

GUICHAOUA A. et GOUSSAULT Y., 2005, Sciences sociales et développement, Paris, Armand Colin.

GUICHAOUA A. et MAJERES J., 1982, " Usages de la sociologie dans les organismes de coopération et du développement ", Revue Tiers Monde, 90 : 423-443.

HANNAH J., 2007, Local Non-Government Organizations in Vietnam: Development, Civil Society and State-society Relations, Ph. D. Dissertation in Geography, University of Washington, Seattle, multigr.

JAULIN R., 1999, Exercices d'ethnologie (ouvrage posthume édité par Roger Renaud), Paris, PUF.

LATOUCHE S., 2003, Décoloniser l'imaginaire. La pensée créative contre l'économie de l'absurde, Paris, Parangon.

LARRERE C. et LARRERE R., 1997, Du bon usage de la nature. Pour une philosophie de l'environnement, Paris, Flammarion.

LAVIGNE DELVILLE P., 1997, «A quoi servent les sciences sociales dans les projets de développement rural ? Points de vue d'un "agent double" ", Bulletin de I'APAD [en ligne].

LAVIGNE DELVILLE P., 2008, "A la recherche du chaînon manquant. Construire des articulations entre recherche en sciences sociales et pratique du développement ", Coopérer Aujourd'hui, 59, GRET.

NAUDET, J.-D., 1999, Trouver des problèmes aux solutions : 20 ans d'aide au Sahel, Paris, OCDE. 
OLIVIER DE SARDAN J.-P., 1995, Anthropologie et développement, essai en socio-anthropologie du changement social, Paris, Karthala.

OLIVIER DE SARDAN J.-P., 2011, "Promouvoir la recherche face à la consultance. Autour de l'expérience du Lasdel (Niger-Bénin)", Cahiers d'études africaines, 202-203 : 511-528.

RAMBO T. A., REED R.R., Le T.C., DI Gregorio M. (eds.), 1995, The Challenges of Highland Development in Vietnam. East-West Center, Honolulu, Hawaii.

RIST G., 2001, Le développement. Histoire d'une croyance occidentale, Paris, Presses de Sciences Po.

\section{Aiviviv}

Emmanuel Pannier est anthropologue, Post-doctorant à l'EFEO (Hanoi),

Chercheur associé au Centre Asie du Sud Est (EHESS, CNRS, INALCO)

E-mail : manuelpannier@yahoo.fr

Christian Culas est anthropologue au CNRS,

Centre Norbert Elias, EHESS (Marseille)

E-mail : christianculas@yahoo.fr 